\title{
Germanica
}

\section{Vom Brennerpass bis Napoli. Heimat, Fremde, Interkulturalität in Kurt Lanthalers Tschonnie- Tschenett-Romanen}

Du Col du Brenner à Naples. Pays natal, étrangeté, interculturalité dans la série Tschonnie-Tschenett de Kurt Lanthaler

Home, abroad, and in between. The intercultural discourse in Kurt Lanthaler's detective novels starring Tschonnie Tschenett

\section{Jürgen Heizmann}

\section{OpenEdition}

Journals

Édition électronique

URL : http://journals.openedition.org/germanica/3213

DOI : $10.4000 /$ germanica.3213

ISSN : 2107-0784

Éditeur

Université de Lille

Édition imprimée

Date de publication : 30 septembre 2016

Pagination : 161-174

ISBN : 9782913857377

ISSN : 0984-2632

\section{Référence électronique}

Jürgen Heizmann, « Vom Brennerpass bis Napoli. Heimat, Fremde, Interkulturalität in Kurt Lanthalers Tschonnie-Tschenett-Romanen », Germanica [Online], 58 | 2016, Online erschienen am: 30 September 2018, abgerufen am 06 Oktober 2020. URL : http://journals.openedition.org/germanica/3213 ; DOI : https://doi.org/10.4000/germanica.3213 


\section{Vom Brennerpass bis Napoli. Heimat, Fremde, Interkulturalität in Kurt Lanthalers Tschonnie-Tschenett- Romanen}

Jürgen HEIZMANN

Université de Montréal

Ein Virus geht um auf dem deutschen Buchmarkt - der Virus des Regionalkrimis. Von Kiel bis Konstanz, von Wismar bis Weinheim wird gemordet, gekidnappt und erpresst. Es gibt auf der deutschen Landkarte kaum noch eine verbrechensfreie Zone. In Österreich und in der Schweiz ist die Lage nicht viel besser, auch dort herrschen Mord und Totschlag auf dem Land. Noch nie gab es so viele ländliche Schauplätze in der Kriminalliteratur wie heute. Joachim Feldmann schreibt dazu in seiner Polemik „Görlitz ist noch krimifrei“, erschienen am 5. März 2011 in der WELT: „Inzwischen wird in fast jedem Provinznest mit Begeisterung gemordet und ermittelt. Je piefiger das Kaff, desto wahnwitziger die fiktiven Morde. Von den exzentrischen Ermittlern gar nicht erst zu reden.“

Dieser Boom des Regionalkrimis, den es in dieser Größenordnung nur im deutschsprachigen Kulturraum gibt, setzte in den achtziger Jahren des 20. Jahrhunderts ein und gewann seither immer mehr an Verve. Von den 700 neuen Krimis, die jedes Jahr in Deutschland erscheinen, haben immer mehr einen regionalen Bezug, ja der Regionalkrimi 
bildet inzwischen ein eigenes Subgenre. Branchenführer ist der Kölner Emons Verlag, der über 50 Regionen Deutschlands in seinem Sortiment hat. Provinz hat im Krimi Konjunktur. Ist dies ein Zeichen für jenen Provinzialismus, den Frank Schirrmacher bereits 1989 an der deutschen Gegenwartsliteratur beklagte1? Mit Sicherheit kann der Beliebtheit globaler Themen und weit entfernter Weltregionen in der deutschen Literatur $^{2}$ ein Hang zum Regionalen an die Seite gestellt werden, eine Ambivalenz, die Thomas Manns Wort vom vereinten „Kosmopolitismus und Provinzialismus im deutschen Wesen" 3 zu bestätigen scheint.

Bis in die siebziger Jahre hinein galt Provinz und alles, was auch nur entfernt an den vorbelasteten Begriff „Heimat“ erinnerte, als rückständig und verstaubt, bieder und beschränkt, antimodern und kitschig. Liebe zum Ländlichen, ein geographisch definiertes, ja womöglich ein freundlich koloriertes Heimatverständnis wurde, vor allem in linksintellektuellen Kreisen, als heikel, ja abstrus angesehen. Zwar begann die Literatur ab den 70er Jahren mehr und mehr, die Provinz zum Thema zu machen, doch in erster Linie, um Deformation, Öde, Fremdheit zu zeigen. Zu Recht wurde in dieser Zeit der Begriff „Anti-Heimatliteratur“ geprägt. Doch in Zeiten der Telekommunikation und der unbegrenzten Mobilität scheint die Bindung an einen Ort das Bedürfnis nach sicheren Positionen und nach einem Identitätsraum zu erfüllen. Die Frage nach der Identität gewann im Zuge der Globalisierung an Dringlichkeit, und die kleine geschlossene Welt einer Region dient in der Literatur oft als Gegenkraft zur Globalisierung und ihrer Phänomene. Das historische Apriori für den gegenwärtigen Provinzroman ist wohl der Fall der Mauer4.

Seither erlebt Regionalismus einen Boom in der Literatur, im Film, in der Werbung und in der Freizeitindustrie. Den erstaunlichen Erfolg des Regionalkrimis muss man in diesem Kontext sehen. Der Genrebegriff verrät das Programm: Der Reiz liegt für die Leser vor allem im Tatort. Natürlich war der Schauplatz der Aktion für Krimis schon immer wichtig. Die Bedeutung der Orte hat in den vergangenen Dekaden aber deutlich zugenommen, sie sind „mehr sogar als die

1. - .Frank Schirrmacher, „Idyllen in der Wüste oder Das Versagen vor der Metropole“, FAZ, 10.10.1989.

2. - Alexander Honold, ,Literatur in der Globalisierung - Globalisierung in der Literatur", www.germanistik.ch, 2010, S.9.

3. - Thomas Mann, „Deutschland und die Deutschen“, in ders.: Reden und Aufsätze 3, Frankfurt/Main, Fischer, 1990, S. 1126-1148, hier S. 1129.

4. - Michael Rölcke, „Konstruierte Enge. Die Provinz als Weltmodell im deutschsprachigen Gegenwartsroman“, in: Carsten Rohde und Hansgeorg SchmidtBergmann (Hg.), Die Unendlichkeit des Erzählens. Der Roman in der deutschsprachigen Gegenwartsliteratur seit 1989, Bielefeld, Aisthesis, 2013, S. 113-138, hier S. 114. 
Detektivfiguren zu Markenzeichen ihrer Serien geworden“"5. Sieht man einmal von Friedrich Glauser ab, lag der Ort des Verbrechens immer in der Großstadt, nun aber ist er in der Nachbarschaft zu finden und ist dem Detektiv in der Regel bestens vertraut. Die anvisierte Lesergruppe wohnt entweder selbst in der Region oder kennt sie von einem Besuch. Dieser Wiedererkennungswert schafft Intimität, der Leser kann sich leicht mit den Figuren identifizieren. Regionale Mentalität und Charaktereigenschaften werden liebevoll ironisch dargestellt und die Orte oft mit Details beschrieben, die für die Plotentwicklung gar nicht wichtig sind. In den Regionalkrimis haben Orte einen erhöhten Eigenwert gewonnen, sie sind selbständige unterhaltende Elemente geworden. Die zahlreichen Lokaldetails sorgen dafür, dass die fiktiven Schauplätze immer eng an die realen Vorbilder bezogen bleiben.

Heimat wird mit Kompensations- und Regenerationsfunktionen verbunden, und das dient faktisch der Stabilisierung bestehender gesellschaftlicher Strukturen. So führen die Verbrechen nie dazu, dass sich die ländliche Idylle als trügerisch erweist. Im Gegenteil: die Krimihandlung scheint kaum in den so positiv dargestellten Ort zu passen und wird denn auch nicht allzu ernst genommen; selbst wenn es um gesellschaftliche Probleme wie Drogenhandel, Korruption oder Umweltverschmutzung geht, wird den Texten nie allzu viel Kritik aufgenötigt. Stattdessen werden immer wieder die Vorzüge und Schönheiten der jeweiligen Region beschworen. Es gibt in den Regionalkrimis oft reiseführerartige Einschübe, die unter Verzicht auf Fiktionalitätssignale und in direkter Kommunikation mit dem Leser Wissenswertes über Land und Leute mitteilen.

Mit dem Regionalkrimi scheint die deutsche Kriminalliteratur, die immer im Schatten der britischen, französischen und amerikanischen Vorbilder stand, ihre Identität gefunden zu haben. Dabei ist das meiste, was unter diesem Etikett in die Buchhandlungen gelangt, banal und läppisch in Hinblick auf Inhalt und Figurenzeichnung, und was den Stil der Romane anbelangt, so verlässt er nie das ausgetretene Terrain des Zeitgeists, der Alltags- und Fernsehsprache, er ist auf leichte Konsumierbarkeit angelegt.

Auch die Autonome Provinz Bozen-Südtirol ist von dem Phänomen Regionalkrimi längst heimgesucht worden. Die Autoren kommen nicht unbedingt aus Südtirol, sondern oft aus Deutschland und Österreich, in jedem Fall aber versuchen die Krimis mit touristischen Informationen das Leserinteresse zu wecken. In Michael Böcklers Tod oder Reben soll in erster Linie die Weinregion Südtirol vorgestellt werden. Die Handlung

5. - Melanie Wigbers, Krimi-Orte im Wandel. Gestaltung und Funktionen der Handlungsschauplätze in Kriminalerzählungen von der Romantik bis in die Gegenwart, Würzburg, Königshausen \& Neumann, 2006, S. 12. 
wird immer wieder durch kleine Abhandlungen zur Geschichte des Weinbaus in Südtirol, zu Rebsorten und zu Anbaumethoden unterbrochen. Die Figuren des Romans üben entweder einen Beruf im Weinsektor aus oder verfügen über einen stattlichen Weinkeller, so dass das Gespräch wie zufällig immer wieder auf Wein kommt. Der Anhang des Romans enthält eine Liste mit Adressen und Beschreibungen von Weingütern, Weinlokalen und Restaurants. Als ,eine ganz spezielle Art von Reiseführer", nämlich einen für Bergtouristen, feiert die Westdeutsche Zeitung Burkhard Rüths Roman Eiszeit in Bozen, der den Leser in die Welt der Dolomiten entführt. Der Prolog bietet eine erdgeschichtliche Abhandlung zur Entstehung der Alpen, deren Gletschwerwelt den Schauplatz der Handlung abgibt. Commissario Vincenzo Bellini, der Held, ist naturgemäß leidenschaftlicher Alpinist und befreundet mit einem in der ganzen Region beliebten Bergführer und Weltenbummler, der in Südtirol jede Berghütte und jede Gletscherspalte kennt. Historisch-kulturelles Wissen hingegen bietet Sigrid Neureiter in dem Roman Kurschattenerbe, der während eines Symposiums über Oswald von Wolkenstein in Meran spielt und allerlei Informationen über den Ritter und Minnesänger sowie über Schloss Tirol enthält. Der Roman bietet eine Karte mit den Schauplätzen der Handlung, einige Neuübertragungen von Liedern Wolkensteins sowie einen Anhang mit weiterführender Literatur zu dem Dichter und zu Schloss Tirol.

Auch wenn in diesen Krimis das Verbrechen in Südtirol eingezogen ist: die völlig isolierte Betrachtung des regionalen Schauplatzes neigt zur Verklärung und zur Verkennung gesellschaftlicher Realität. Es gehört zum Trenddesign dieser Krimis, dass die ländliche Region Hort der Treue und Unschuld bleibt. Die Verbrecher sind darum auch immer Ausnahmefiguren und schon beruflich eher dem urbanen Milieu zugehörig: Der Täter in Tod oder Reben ist ein arroganter Schönheitschirurg; in Kurschattenerbe ist es ein von krankhaftem Ehrgeiz getriebener Mediävist; in Eiszeit in Bozen schließlich ein pathologisches Monster, das quasi aus dem Nichts auftaucht und im Nichts verschwindet. Die Verbrecher sind nie typische Vertreter der einheimischen Bevölkerung und sprechen darum auch nie Dialekt wie viele andere Figuren.

Kurt Lanthaler ist mit seinen fünf Romanen um den Ermittler Tschonnie Tschenett der weithin bekannteste Südtiroler Autor in diesem Genre - sein Serienheld hat es immerhin als einer der wenigen deutschsprachigen Vertreter in Heiko Postmas Galerie der Detektive 6 geschafft. Lanthaler untergräbt aber zugleich die Regeln des Genres, denn seine Romane gehen weit über die Konventionen des Krimis

6. - Heiko Postma, Galerie der Detektive. 123 Portraits von Sherlock Holmes bis Nero Wolfe, Hannover, Revonnah, 1997. 
hinaus und entwickeln einen komplexen Heimatbegriff, der die Reiseführermentalität des Regionalkrimis verspottet.

Lanthaler, 1960 in Bozen geboren, gehört zu jener Generation Südtiroler Autoren, die mit Heimatdichtung und Heimattümelei abrechneten, die Literatur nicht mehr als Kampf und Selbstbespiegelung der deutschsprachigen Südtiroler auffassten. Zu diesem Aufbruch junger Südtiroler Autoren schreibt Siegrun Wildner:

Während die HeimatliteratInnen in ihren Werken auf Schutz, Festigung und Ausbau einer kollektiven ethnischen Identität der deutschsprachigen Bevölkerung setzten, forderte die neue Generation eine Öffnung nach Außen und ein ethnisches Miteinander, besonders mit der italienischen Volksgruppe in Südtirol. Die neuen AutorInnen brachen mit den Tabuthemen der Heimatliteratur und setzten sich kritisch mit Faschismus und Nationalsozialismus, mit der Frage der Option und der deutschen Besetzung Südtirols zwischen 1943 bis 1945, mit den Monopolstrukturen und dem ausgeprägten Katholizismus in Südtirol auseinander?

In Tschonnie Tschenetts Südtirol begegnen uns denn auch Neofaschismus, Spekulantentum und Korruption. Die fünf Romane Der Tote im Fels, Grobes Foul, Herzsprung, Azzurro und Napule werben auf dem Cover nicht mit dem Etikett „Südtirol-Krimi“. Stattdessen wird auf die Serie verwiesen: ein Tschonnie-Tschenett-Roman. Nur die Erstausgaben der beiden ersten Romane führten zudem die Gattungsbezeichnung „Kriminalroman“, ab dem dritten Band und in den Neuausgaben fiel sie weg.

In dem Roman Herzsprung findet sich eine poetologische Autoreflexion, wie Lanthaler dazu kam, Kriminalromane zu schreiben. Er zitiert, leicht abgewandelt, einen Satz aus Friedrich Glausers Roman Der Tee der drei alten Damen: „Spotte mir nicht über Kriminalromane! Sie sind heutzutage das einzige Mittel, vernünftige Ideen unter die Leute zu bringen“ (Herzsprung, S. 271.) Das spielt auf jenen Zweig der Kriminalliteratur an, der sich als Fortsetzung des realistischen Romans versteht. Gemeint ist also nicht die Tradition der britischen Lady of Crime Agatha Christie mit ausgeklügelten Morden auf gepflegten Herrensitzen, sondern Geschichten über eine tatsächlich existierende Welt, in der reale Menschen reale Verbrechen begehen und Schmerzen haben und Schuld sühnen. Ich sehe Lanthalers Serie darum auch als plebejische Antwort auf die Venedig-Krimis der Amerikanerin Donna Leon, die auf ein bildungsbürgerliches Publikum zugeschnitten sind.

7. - Siegrun Wildner, „Einleitung: Die Neuere Literatur aus Südtirol“, in dies. (Hg.), (W)orte.Zeitgenössische Literatur aus und über Südtirol, Innsbruck-Bozen-Wien, Skarabaeus, 2005, S. 13-67, hier S. 21. 
Donna Leons Romane um den Commissario Brunetti stehen ganz in der Tradition des beschaulichen, altmodischen angelsächsischen Krimis, nur haben sie ihn an einen neuen Schauplatz verlegt. Ihre Romane sind Märchen, die mit dem realen Italien nichts zu tun haben und wohl verantwortlich sind für eine ganze Reihe von Italien-Krimis in diesem Strickmuster.

In einer Gesprächsrunde mit verschiedenen deutschsprachigen Krimiautoren macht Lanthaler deutlich, dass die Möglichkeiten und Chancen des Kriminalromans für ihn vor allem mit der Rezeption und dem Literaturbetrieb zu tun haben. Da der Kriminalroman im deutschen Raum im Feuilleton, auf welches andere Romane schielten, kaum wahrgenommen werde, böte er auch mehr Freiheiten, wenn man sich präzise mit Zuständen und Personen befassen wolle ${ }^{8}$. Dies ist ein Hinweis auf die geltenden Regeln im System der Künste, auf das bourdieusche Feld. Ist ein Genre wie der Kriminalroman in der Hierarchie eher unten angesiedelt, eröffnet das größere Spielräume. Lanthaler nutzt das Genre zu seinen Zwecken: Faktengenauigkeit in ökonomischen, politischen und sozialen Zusammenhängen zu bieten, doch zugleich wandelt er das Genre ab. Worin bestehen nun die Abweichungen vom Genreüblichen?

Außergewöhnlich an der Tschonie-Tschenett-Serie ist das umfangreiche Glossar, das jedem Roman angehängt ist. Im Gegensatz zu den vergleichbaren Paratexten in herkömmlichen Regionalkrimis beschränkt es sich keineswegs auf touristische Informationen, auch wenn sich gelegentlich ein Kommentar zu Südtiroler Orten, Begriffen oder ein Rezept der einheimischen Küche findet. Lanthaler übernimmt solche Elemente, aber meist um sie zu ironisieren und zu sabotieren. So findet man in Herzsprung Wandervorschläge, die sich aber keineswegs auf Tschenetts touristisch beliebte Heimatregion Südtirol beziehen, sondern auf die Umgebung der 320-Seelen-Gemeinde Herzsprung, die sich irgendwo im Niemandsland zwischen Rostock und Wittstock befindet, und die Wandervorschläge sind einer 1975 in der DDR verlegten Broschüre entnommen (S. 274). Während die Geschichten von der Figur Tschenett erzählt werden, meldet sich in dem Glossar der Autor selbst zu Wort und vertieft verschiedene Aspekte des Erzählten, liefert historische Details und zieht aktuelle politische Nachrichten heran. Im Wechselspiel mit der Romanhandlung ergibt sich daraus eine virtuose Mischung aus Fakten und Fiktion. Umfang und Bedeutung des Glossars nehmen im Verlauf der Serie zu, in Napule macht es beinahe ein Fünftel des Romans aus. Dort legt Lanthaler die informativen, oft auch sarkastisch gefärbten Erläuterungen und Ergänzungen seinen Protagonisten in den Mund und integriert sie damit in die Handlung, macht sie zum Teil der fiktionalen

8. - Nils Jensen, „Ritual \& Mord. Wie ist das mit dem Krimi? Interviews“, in: Buchkultur 39.3 (1996), S. 32-39, hier S. 32. 
Geschichte. In Napule erprobt Lanthaler aber auch schon jene Form, die er später Glossom nennen wird. Dies ist eine Wortneubildung aus Glossa und Rhizom und bezeichnet eine Art der Fortschreibung, die im Sinne von Gilles Deleuze und Félix Guatarri Querverbindungen, Verzweigungen, Überschneidungen und perspektivische Verschiebungen herstellt. Diese Schreibweise versucht dem ehernen Gesetz der Linearität zu entkommen, sie ermöglicht ein herkömmliches, lineares, aber auch ein thematisch springendes, dekompositorisches und transformatorisches Lesen. Sie versucht, der Komplexität und der Kontingenz der Wirklichkeit, die Lanthaler beschreibt, gerecht zu werden. Der Leser kann das Gedankengebäude auf seinem eigenen Weg, wie immer es ihm sinnvoll erscheint, durchschreiten.

Auf seiner offiziellen Homepage schreibt Lanthaler mittlerweile die Glossare zu den Tschonnie-Tschenett-Romanen in Form solcher Glossome weiter und bietet auch Glossome zu noch nicht geschriebenen Romanen, so dass es, wie es im Geleitwort seiner textverarbeitenden Manufaktur heißt, am Ende keines Romans mehr bedarf ${ }^{9}$.

Schon für die Neuausgaben der Romane wurden die Glossare erweitert und aktualisiert. Gelegentlich greift Lanthaler dabei auch Anregungen von Lesern auf, so dass in diesen Paratexten eine Art interaktive Literatur entsteht.

Der Protagonist Tschonnie Tschenett ist ein Südtiroler, den es als junger Mann nicht länger in der Enge seiner Heimat gehalten hat. Ende der sechziger Jahre ist er nach Hamburg aufgebrochen, hat auf einem Fischkutter angeheuert und war viele Jahre als Matrose auf der Nordsee unterwegs. Zur Handlungszeit in Der Tote im Fels ist Tschenett seit fünf Jahren wieder zurück aus seinem freiwilligen Exil. Gleich zu Beginn fragt er sich, ob diese Rückkehr nicht ein Fehler war. „Nach den vielen Jahren im hohen Norden. Bis es mir dort zu kalt geworden war. Und ich Richtung Süden gezogen war. Und dort auch keine richtige Wärme gefunden hatte. Weiter südlich war es mir zu heiß, und hier, mittendrin, hielt ich es nicht aus.“(S. 25) Lanthalers Romane decken keineswegs die Bedürfnisse nach Erdung in der Heimat, nach einem Ort wohliger Ruhe in Zeiten der Beschleunigung und Globalisierung. Ihm ist sogar im scheinbar beschaulichen Südtirol zu viel los. „Denn was sich da um mich herum abspielte an Geschäftigkeit, Tüchtigkeit, Fleiß und Pünktlichkeit und wie diese Kardinaltugenden einer verrottenden Welt alle hießen, konnte einem die Pickel bis ins letzte Glied schießen lassen“ (Grobes Foul, S. 19).

Schon hier wird deutlich: Tschenett ist kein typischer Einheimischer mit ein paar kauzigen Eigenschaften, wie wir ihn in den meisten

\footnotetext{
9. - Siehe www.homepage.hispeed.ch/lanthaler/.
} 
Regionalromanen als Serienhelden antreffen. Er ist ein Unangepasster, der im Clinch mit der bürgerlichen Ordnung liegt, die er als Unordnung begreift. Er arbeitet als Aushilfsfahrer bei verschiedenen Speditionsfirmen (die ihre Fahrer skrupellos ausbeuten und regelmäßig übers $\mathrm{Ohr}$ zu hauen versuchen), ist also ein underdog, und sein Blick auf die korrumpierte Gesellschaft ist ein Blick von unten - nach Thomas Wörtche ist dies der Blick, den echte Kriminalliteratur $\mathrm{zu}$ haben hat ${ }^{10}$. Ob es um Schlepperbanden geht, die Menschen in Kühllastern nach Italien schleusen, um Zigarettenschmuggel in Millionenhöhe, an dem die Tabaklobby selbst dann noch verdient, wenn die illegale Ware auffliegt, ob es um Schmiergeldzahlungen Silvio Berlusconis an die Guardia di finanza geht oder um den weltberühmten Tiroler Speck, der mit holländischen Schweinehälften produziert wird: für Tschenett ist die Wirklichkeit chaotisch, irrwitzig, gewalttätig und sinnfrei, beileibe kein wohlgeordneter und wohlfunktionierender Ort. Die Antwort darauf ist Komik, eine Komik voll Wut, Aggression und Resignation. Die Erzählhaltung in den Romanen ist mit der des jungen Arno Schmidt vergleichbar: Wie bei Schmidt gibt es bei Lanthaler die Respektlosigkeit, das Antiautoritäre, das ni dieu ni maître des Erzählers und sein bitter-höhnisches Lachen angesichts der Härten des Daseins. Tschenett ist denn auch kein Saubermann, kein makelloser Serienheld. In Herzsprung wird er unwissentlich selbst in den Zigarettenschmuggel hineingezogen, macht dann aber willentlich mit. Das Gute, heißt es in Azzurro, ist nur ein anderes Wort für Dummheit (S. 62). Der brave Mann denkt an sich selbst zuerst, lautet gewissermaßen die Devise. Erst als Tschenett bemerkt, dass beim Schmuggelgeschäft Nordvietnamesen, die einst von der DDR als Vertragsarbeiter angeheuert wurden und nun illegal in Deutschland leben, ausgebeutet und umgebracht werden, sowie sie aufbegehren, regt sich sein Gewissen.

Allein dadurch, dass er auf den Straßen Europas unterwegs ist, kommt bei Tschenett auch immer wieder der Gedanke auf, seine erste Heimat für immer zu verlassen. Eine Kontrastfigur zu ihm stellt die 65 Jahre alte Wirtin Berta dar. Im Gegensatz zu dem ziellosen Weltenbummler ist die verwitwete Berta nie aus Südtirol, ja nicht einmal aus ihrem Tal herausgekommen. Sie betreibt eine winzige Bar direkt an der Straße im Pflerer Tal, im Grenzgebirge zwischen Italien und Österreich. Obwohl Berta fest in Südtirol verwurzelt ist, ist auch sie eine Außenseiterin, denn sie betreibt ihre Bar ohne Lizenz oder Genehmigung: „Sie schenkte einfach aus. Bis jetzt hatte ihr noch niemand einen Strick daraus gedreht. Was bei der Geiermentalität, die manche Leute umtreibt, beinahe schon an ein Wunder grenzte“ (Der Tote im Fels, S. 19-20). Für den respektlosen

10. - Thomas Wörtche, Das Mörderische neben dem Leben. Ein Wegbegleiter durch die Welt der Kriminalliteratur, Lengwil (CH), Libelle, 2008, S. 22. 
Tschenett, der sich mit beinahe jeder Art von Autorität anlegt, ist Berta die einzige Respektperson.

Die dritte wichtige Figur ist der italienische Polizist Totó, einer der Nachbarn Tschenetts, mit dem er sich langsam anfreundet, denn auch Totó ist ein Außenseiter. Er ist zwar in Südtirol geboren und hat eine deutsche Mutter, doch er fühlt sich in der Alpenprovinz oft fremd. Sein Vater stammte aus Apulien, im Faschismus hatte es ihn durch ,unglückliche Zeitumstände" nach Alto Adige verschlagen. Totó ist bei seinen Kollegen nicht sehr beliebt, denn er hält es im Gegensatz zu ihnen nicht mit Liebdienerei und Karrieredenken, auch wenn ihm das natürlich schadet. Am Ende des zweiten Bandes wird er „nach Sibirien“, d.h. auf den Brennerpass verbannt, um am Grenzübergang Autos durchzuwinken. Er trägt Tschenett Informationen aus dem Inneren des Polizeiapparates zu, die diesen oft etwas schneller sein lassen als die Behörden.

Im ersten Band der Serie, Der Tote im Fels, wird ein Toter aus dem Geröll einer Tunnelbaustelle im Pflerer Tal geborgen. Bald wird an der Eisenbahnstrecke nach Österreich ein zweites Mordopfer gefunden, und der Chef eines norditalienischen Sondereinsatzkommandos erscheint in Südtirol. Tschenett und Totó kommen dahinter, dass es bei den Verbrechen um den Bau des Brennerbasistunnels geht und um damit in Zusammenhang stehende Grundstückspekulationen. Der Bau eines Eisenbahntunnels, der tief unten im Berg den Brenner (die wichtigste Nord-Süd-Verbindung in Europa) unterlaufen soll, war in der Tat schon seit den sechziger Jahren in Planung, doch wegen politischer Intrigen ist er immer wieder verschoben worden: „Das Problem war dabei vor allem, daß der Brennerpaß zugleich auch Grenzübergang war. Und nicht nur irgendeiner. Sondern ein tirolischer, und ein italienischer, und wer weiß was noch. Und einen solchen Grenzübergang einfach so zu untertunneln, war anscheinend nicht möglich“ (Der Tote im Fels, S. 10).

Auch wenn seit dem Schengener Abkommen die Grenzkontrollen aufgehoben wurden, steht der Brennerpass immer noch unter strenger Bewachung und hat de facto zu einer erheblichen Ausweitung polizeilicher Befugnisse geführt. 2016 drohte Österreich, am Brenner wieder Kontrollen einzuführen, um Flüchtlinge abzuhalten. Mit dem Bau des Brennerbasistunnels wurde inzwischen zwar begonnen, über den genauen Verlauf wird aber immer noch zwischen Italien und Österreich debattiert und wann er fertig gestellt wird, steht in den Sternen. Im Roman finden Tschenett und Totó heraus, dass sowohl italienische als auch deutsche und österreichische Nationalisten den Tunnel zu verhindern suchen, obwohl dieser von der EG befürwortet wurde und die Politiker aller drei Nationen in ihren Sonntagsreden den Brennerbasistunnel als Jahrhundertwerk und als Symbol für das Europa von morgen feiern. Doch hinter den Morden stecken nicht nur 
politische, sondern auch wirtschaftliche Interessen. Die Spur führt zur Tiroler Investment, einer Abschreibefirma, die es der haute volée Bozens, und zwar der deutschen wie der italienischen, ermöglicht, nicht ganz eindeutig erworbene Gelder am Staat vorbei ins Trockene zu bringen. Die Tiroler Investment arbeitet zusammen mit einem Innsbrucker Ingenieurbüro, das vor allem in die eigenen Taschen wirtschaftet. Der Direktor dieses Büros ist Klaus-Dieter Pressack, ein angesehener Unternehmer trotz seiner nationalsozialistischen Vergangenheit. Pressack war nach dem Anschluss Österreichs an Nazideutschland daran beteiligt, die Universität Innsbruck nationalsozialistisch auszurichten. Sein Vorgesetzter bei dieser Aktion - und hier baut Lanthaler recherchierte Fakten ein - war der SS-Hauptsturmführer Hanns Martin Schleyer, der in der Bundesrepublik Präsident des Bundesverbands der deutschen Industrie (BDI) und der Bundesvereinigung deutscher Arbeitgeberverbände (BDA) war und 1977 von einem Kommando der RAF entführt und erschossen wurde. Die Figur Pressack führt aber auch in ein schwieriges Kapitel der Südtiroler Geschichte zurück, denn er engagierte sich in den sechziger Jahren für eine Stärkung des deutschen Volkstums in Südtirol und unterstützte die sogenannten dinamitardi, die Bombenleger in Südtirol, mit Sprengstofflieferungen, was ihm die italienischen und österreichischen Behörden jedoch nie nachweisen konnten, zumal Pressack auch gute Kontakte zu italienischen Neofaschisten besitzt.

Vieles deutet darauf hin, dass Pressack hinter den Morden steht, da er unliebsame Mitwisser seiner Machenschaften aus dem Weg räumen lassen musste. Doch ein Mann mit solchen Verbindungen ist für Tschenett und Totó eine Nummer zu groß; am Ende müssen sie froh sein, ungeschoren davonzukommen. Die Schlussszene zeigt Pressack in einem Südtiroler Festzelt. Grinsend lädt er Tschenett zu einem Bier ein. Alles, was dem Gelegenheitsdetektiv als Geste des Protests bleibt, ist, die Einladung auszuschlagen.

Plötzlich hatte ich es eilig. Draußen vor dem Festzelt lehnte ich mich gegen eine Mauer und kotzte.

„Da sieht die Welt gleich viel schöner aus“, sagte neben mir einer, der es gerade hinter sich gebracht hatte.

„Das ist die Welt", sagte ich und schaute auf das, was da vor mir auf dem Boden lag. (Der Tote im Fels, S. 244)

Der Tote im Fels ist ein Kriminalroman ohne Lösung. Der Detektiv kommt den Drahtziehern des Verbrechens zwar auf die Spur, doch die Schuldigen gehen straffrei aus. Das einzige, was sich am Schluss geändert hat, ist, dass Tschenett nun noch mehr entschlossen ist, seine Heimat wieder zu verlassen. Diese pessimistische Weltsicht widerspricht dem prononciert optimistischen Erzählschema des konventionellen Krimis, 
auch und gerade des Regionalkrimis. Das organisierte Verbrechen ist in die normale gesellschaftliche Ordnung integriert, so dass der Detektiv mit seinen Untersuchungen die Ordnung stört. Dringt er zu weit ins Zentrum vor, wehrt sich das System mit Macht: Der Detektiv bleibt Außenseiter und Störenfried. Rückkehr in die Normalität bedeutet jetzt Rückkehr ins Verbrechen. Auch im Roman Herzsprung, der den EG-weiten Zigarettenschmuggel zum Hintergrund hat, kann Tschenett gegen das Verbrechen, gegen das System aus Korruption und Betrug bei Tabakkonzernen, Speditionsfirmen und Zollbeamten nichts ausrichten. Er kann lediglich das Leben zweier Nordvietnamesen retten, indem er sie mit falschen Pässen über den Brenner nach Südtirol schmuggelt.

Dieser kritische Rekurs auf den Kriminalroman verrät den Einfluss des sizilianischen Erzählers und Essayisten Leonardo Sciascia, den Lanthaler selbst als eines seiner Vorbild nennt ${ }^{11}$. Sciascia bietet den Lesern in seinen Krimis, in der es in der Regel um die Mafia geht, ebensowenig eine befriedigende Lösung an. So deckt der Detektiv in Il giorno della civetta zwar die Verbindungen zwischen der Mafia und einem Bauunternehmen auf, doch die Verbrecher haben Verbindungen zu Parlamentsabgeordneten in Rom und bleiben von der Justiz verschont, während der unliebsame Detektiv seinen Posten verliert.

Weder bei Sciascia noch bei Lanthaler gibt es eine plötzliche überraschende Wendung. Keine einzelne, dämonisierte Figur ist für das Verbrechen verantwortlich, sondern das System. Dieses kriminelle System ist gesellschaftlich etabliert und kaum noch ein Geheimnis, das zu entdecken wäre. Es gibt eine Konvergenz von Verbrechen und Staat. Die Aufklärung eines Verbrechens spielt darum im Lauf der Serie eine immer weniger bedeutende Rolle; damit zusammenhängend weicht die zielgerichtete Plotentwicklung ab Herzsprung einer episodischen Struktur des Erzählens. Auch der Schauplatz Südtirol gerät ab dem dritten Band der Serie in den Hintergrund, große Teile der Handlung geschehen nun an anderen Orten, in Berlin, in Albanien oder in Neapel. Im letzten Band der Reihe kommt die Alpenegion gar nicht mehr in den Blick, Tschenett hat sich mittlerweile in Griechenland niedergelassen, einen echten Fall gibt es in Napule nicht mehr, eher die wehmüitige Poesie einer Stadt der Lust und des Leidens. „Tatsächlich“, stellt der neapolitanische Hilfspolizist Ciro resigniert fest, ,gibt es das große, kapitale Verbrechen nicht mehr, spätestens seit Andreotti freigesprochen, Berlusconi verjährt und der eine oder andere Faschist an der Regierung ist" (Napule, S. 48).

Wenn es in keinem der Romane ein echtes Happy End gibt, darf man fragen, warum sich dieser Tschonnie Tschenett überhaupt engagiert. Es

11. - „Interview mit Kurt Lanthaler“, in: http://www.buchkritik.at/autoren/ lanthaler.htm. 
liegt wohl daran, dass er weiß, dass überall die kleinen Leute von den Kapitalisten aufgesogen werden und die Welt vor moralischem Verfall nur so stinkt. Südtirol erscheint als eine Region, die wie immer schon zugleich Spielball und Schauplatz verschiedener nationaler Interessen ist. Dabei stellen die Romane Südtirol als ein Gemisch aus zwei Kulturen dar. Es ist längst nicht mehr Österreich, aber auch noch nicht ganz Italien. Auch sprachlich bewegt sich der Protagonist zwischen den beiden Kulturen, es gibt sehr viele italienische Einsprengsel, aber auch sehr viele Südtiroler Dialektausdrücke; in dem Roman Herzsprung kommt auch das Ladinische hinzu.

Auch wenn Lanthaler seine Romane auf Schriftdeutsch schreibt, spielen diese dialektalen und italienischen Elemente eine ganz wichtige Rolle; sie werden bedeutsam wie Namen. Oft sind es nur einzelne Wörter, Floskeln und Redewendungen, die in die Rede eingefügt werden. In ihnen verdichtet sich jedoch Heimat. In Grobes Foul erinnert sich Tschenett, warum er nach zwanzig Jahren Exil in Deutschland plötzlich wieder in Südtirol hängenblieb: nämlich dass man dort einen Satz auf Deutsch beginnen und auf Italienisch zu Ende führen konnte (S. 71). Tschenett plädiert für einen offenen, auf Austausch angelegten Kulturbegriff, ebenso wie sein Freund Totó, der als einziger der vielen italienischen Polizisten ausgezeichnet Deutsch spricht. Beide sind offen für das Fremde, das Andere, sehen es als eine Möglichkeit der Entwicklung und Selbstverwirklichung. Das Ungemütliche an der Heimat rührt daher, dass viele der Bewohner sich gegen die andere Kultur abschotten. Tschenett stellt fest, dass die Menschen in dieser italienischen Provinz in den Bergen zwar ,in drei Sprachen redeten, aber immer nur von einer die Rede ging. Von der eigenen." (Grobes Foul, S. 25) Der Katholizismus, den viele deutsche Bewohner noch als identitätsstiftendes Bindemittel betrachten (der italienische Feiertag ferragosto ist für die deutschstämmigen Tiroler Hochunserfrauentag, an dem man bündelweise Kräuter in die Kirche trägt, um sie segnen zu lassen), dieser Katholizismus ist für Tschenett nur Bigotterie. Die Gefahr für die Heimat kommt gerade von jenen, die eine dezidierte Heimatideologie vertreten, eine Ideologie, die von Nazi-Erbe durchsetzt ist und durch Abgeschlossenheit und Ignoranz gegenüber jeder anderen Perspektive geprägt ist. Alles was an Brauchtum erinnert und in herkömmlichen Regionalkrimis gern als Urtümlichkeit gefeiert wird, ist Tschenett darum gründlich verhasst, zumal er durchschaut, dass die geschäftstüchtigen Südtiroler es benutzen, um damit Touristen anzulocken, die auf der Suche nach dem Echten, Wahren, Unverfälschten sind. Ein Beispiel dafür ist der Dekor des Försterkellers, laut Tschenett eine „Diskothek für Volksmusikfanatiker“: 
Rundherum Holz, soweit das Auge trug. Höchst schlampig auf alt gemachte Baumstämme, die an die Betondecke gedübelt worden waren. Eine Sitzecke, die so tat, als sei sie eine Almhütte. Rotweißkarierte Vorhänge. Hörner und Felle diverser alpiner Gattungen an der Wand. Und die abscheulichsten Blech-, Messing- und Eisengerätschaften, die man sich nur vorstellen konnte. Eine Folterkammer. (Grobes Foul, S. 25-26)

In dieser „Folterkammer“ kommt es dann auch immer wieder zu Schlägereien zwischen den Tiroler Jungbauern aus den verschiedenen Seitentälern und den hier stationierten italienischen Soldaten, die ihren Militärdienst ableisten müssen.

Ungemütlich ist die Heimatregion aber auch durch die Arroganz der italienischen Ordnungshüter, die alle Südtiroler wie tumbe Bauern behandeln. Tschenett verwendet für sie das in Italien verbreitete Schimpfwort romanaccio, mit dem man die Hauptstädter bezeichnet. In dem Ausdruck sind alle Untugenden zusammengefasst, die in der Zentrale eines zentralistischen Staates zu finden sind, in erster Linie Überheblichkeit und parasitäres Verhalten. Die zahlreichen italienischen Ausdrücke sind wichtig für die dargestellte Sprachheimat.

Tschenetts Offenheit zeigt sich auch an seinen beiden besten Freunden: eine alteingesessene deutsche Südtirolerin und ein heimwehkranker Italiener mit Wurzeln in Apulien. Zieht es Tschenett ständig über die Brennergrenze in Richtung Norden, so seinen Freund Totó in den tiefsten Süden Italiens, während Berta meint, sie käme um, wenn sie die Heimat verließe. Am Ende des Romans Azzurro wird Totó so apathisch und krank, dass nur noch eine Reise in seine väterliche Heimat helfen kann. Auf der Fahrt in den Süden sitzt Totó unbeweglich und schweigsam da, bis er auf der Höhe von Mantova auf einmal den Satz sagt: „Miaßn, Hofer, tian mir gor nicht“ (S. 291). Eine Anspielung auf den in deutschnationalen Kreisen noch immer verehrten Andreas Hofer, den Anführer der Tiroler Aufständischen gegen die bayrisch-französische Besatzung unter Napoleon, für Tschenett aber auch ein Aufstand gegen die Aufklärung. Als Hofer die Chefs der Schützenkompanien zusammenrief, um sie zu einer letzten und aussichtslosen Schlacht aufzurufen, stand einer der Bauern auf und sagte: „Miaßn, Hofer, tian mir gor nicht.“ Titó, der Sohn apulischer Kleinbauern, kann sich mit dem Eigensinn der Tiroler Bauern identifizieren, spiegelt sich darin doch auch der Eigensinn, den er gegen die italienischen Autoritäten zu bewahren sucht. Die Erinnerung an diesen Spruch wirkt darum wie eine Befreiung.

Eine Heimat stiftende Rolle wie die Sprache spielen in Lanthalers Romanen die Speisen und Getränke. Es finden sich in den Romanen Rezepte für Knödelbrot, für polenta und für Tirteln, einer ladini- 
schen Spezialität, mit der Tschenett einmal Totó und einen befreundeten italienischen Fußballer bewirtet. Deren Begeisterung zeigt deren Aufgeschlossenheit für das Neue und Fremde. Tschenett schwärmt ebenso vom Lagrein, dem berühmten Rotwein Südtirols, wie vom Gravina, einem apulischen Weißwein. Hier scheint Lanthaler der Reiseführerästhetik des Regionalkrimis nahe zu kommen. So weist er im Glossar zu Azzurro auf ein kleines, exzellentes Speiselokal hin, das man in der Fischergenossenschaft eines apulischen Ortes finden könne. Im Gegensatz zu den konventionellen Regionalkrimis verrät Lanthaler jedoch nicht alles: „Die Adresse des Lokals können Sie beim Verlag nicht erfragen, auch nicht, wenn Sie Rückporto beilegen. Das Lokal ist zu klein, um es schadlos den Massen zu verraten“" (S. 324).

Diese Haltung entspricht dem herrschenden Heimatbegriff in der Romanserie. Heimat gibt es nur als Ort des Privaten in einer Welt des Tourismus, der Anonymität und des nivellement. Heimat gibt es auch nicht als festen Besitz, sie muss immer wieder neu erworben werden. Das Fernweh Tschenetts ist darum auch eine Art Heimweh. Dem letzten Roman der Serie, Napule, ist fogerichtig das Motto eines neapolitanischen Liedes vorangestellt: Die Schiffe laufen / zu fernen Ländern aus. 